\title{
Effect of nutritional stress on the tsetse fly's vector competence and its implications on trypanosome transmission in the field
}

\author{
Gilbert Komlan Akoda \\ Supervisors: Pierre Dorny (1), Peter Van den Bossche (2), \\ Jan Van Den Abbeele (2)
}

(I) Department of Virology, parasitology and immunology, Ghent University

(2) Institute of Tropical Medicine, Antwerp

Public defence: 17 December 2009

The obligate blood feeding tsetse fly (Diptera: Glossinidae) is an essential component in the cyclical transmission of several African trypanosome species such as Trypanosoma congolense and T. brucei spp., causing devastating diseases in both humans and animals in sub-Saharan Africa. The proportion of trypanosome-infected tsetse is a major determinant of the transmission dynamics of the disease and it is affected by various endogenous and exogenous factors. Recently, the nutritional state of the flies at the time of their infective bloodmeal was demonstrated to affect the susceptibility of teneral and older flies to infections with T. congolense or T. b. brucei. The experimental work that is presented in this thesis aimed i) to determine whether nutritional stress affects the ability of tsetse flies to develop human pathogenic trypanosome species, ii) to explore the underlying mechanism that induce an increased susceptibility in starved tsetse flies with focus on the tsetse fly's immunological abilities, and finally iii) to determine the effects of seasonal climatic changes on the nutritional status and immune peptide expression in field-caught tsetse flies, in order to determine whether changes in environmental conditions affect the trypanosome transmission dynamics in the field.

The first chapter of the thesis reviews our current knowledge on the endogenous and exogenous factors that affect tsetse-trypanosome interactions with special attention to tsetse-related factors that interfere with the parasite midgut establishment and maturation in the insect vector.

In chapter 2, starved and non-starved teneral and older flies were experimentally infected to determine the effect of starvation on their susceptibility to T. b. gambiense or T.b. rhodesiense. Use was made of tsetse species belonging to the savannah (G. m. morsitans) or the riverine subgenera (G. p. gambiensis) in order to mimic the field situation as much 
as possible. The results of this study revealed that starvation occurring before the infective bloodmeal increases the intrinsic vectorial capacity of tsetse flies for $T . b$. rhodesiense by lowering the developmental barrier especially at midgut level. Several experimental infections using different T. b. gambiense strains were unsuccessful due to a very low ability of the parasites to establish in the tsetse fly's midgut. This demonstrates the difficulty in obtaining a suitable T. b. gambiense-Glossina experimental model that allows the study of factors influencing this parasite's development.

In chapter 3, a study determining the effect of starvation of tsetse flies on the maturation of a $\mathrm{T}$. brucei procyclic midgut infection into a metacyclic salivary gland infection is presented.

Tsetse flies, infected as teneral, were starved for seven consecutive days ro days after the infective bloodmeal. Results of this experiment showed a significantly increased proportion of flies with salivary gland infection in the nutritionally-stressed fly group, suggesting an enhanced maturation T. b. brucei infection due to the nutritional stress during the critical period of salivary gland colonization.

In the fourth chapter, the effect of nutritional stress experienced by adult reproducing female tsetse flies on their offspring's susceptibility to trypanosome infection is determined. The results of this study revealed a significant increase in the intrinsic vectorial capacity, for infections with T. congolense or T. b. brucei, of teneral flies emerging from starved adult females happened more frequently compared to those emerging from non-starved females. This suggests that in the field situation, environmental conditions that nutritionally stress adult reproducing female tsetse flies may not only affect their reproductive performance but also increase the number of infected flies that emerged from pupae produced by these stressed females.

Chapter 5 investigates the effect of nutritional stress on i) the non-induced baseline gene expression level of the antimicrobial peptides attacin, defensin and cecropin in the tsetse fly and ii) their induced expression level in response to bacterial (E. coli) or trypanosomal challenge. Results show that starvation of newly hatched, unfed tsetse flies significantly lowers their baseline antimicrobial peptide expression level especially for attacin and cecropin. In response to trypanosome challenge, only non-starved older flies showed a significant increase of the antimicrobial peptide gene expression within 5 days after ingestion of a bloodmeal containing trypanosomes, especially so for $\mathrm{T}$. brucei bloodstream forms. These data suggest that a decreased immune gene expression level in newly hatched flies or a lack of immune responsiveness of older flies to trypanosomes, as a result of fly starvation, could be one of the contributing factors to the increased susceptibility of nutritionally-stressed tsetse flies to a trypanosome infection.

Chapter 6 presents a study conducted in the Zambezi Valley (Zimbabwe) during the hot dry season (corresponding to a period of increased nutritional stress for tsetse flies) and the rainy season (corresponding to a period of lower nutritional stress for tsetse flies). The study aimed at determining seasonal changes in the nutritional and immune status of tsetse flies with a possible impact on the trypanosome transmission dynamic in the field. The results show that the nutritional state and the expression levels of anti- 
microbial peptides attacin, defensin and cecropin are reduced during the hot dry season compared to the rainy season, especially in G. m. morsitans. However, the possible repercussions of such variation on trypanosome transmission by this tsetse species were difficult to assess due to the limited number of tsetse caught and dissected, which did not allow the establishment of an age-infection prevalence relationship that would highlight changes in the susceptibility of especially adult flies.

In the chapter 7, we investigate the effect of the developmental stage of a monomorphic $T$. congolense strain in the mammalian host on its transmissibility by the tsetse fly. The results showed that the development stage of the trypanosome in the host blood does affect the proportion of flies that develop a mature or immature infection with significantly higher infection rates in flies that were fed on days 5 or Io post-infection of the mammalian host (mouse). These findings stress the importance of standardizing experiments in which the vectorial capacity of tsetse flies is determined and compared.

In the final general discussion (chapter 8), the major findings of the thesis are discussed in the wider context of the possible impact of nutritional stress and environmental stressors on the epidemiological situation of sleeping sickness. 